\title{
In Vitro Biological Evaluation of Electrospun Polycaprolactone/Gelatine Nanofibrous Scaffold for Tissue Engineering
}

\author{
Mim Mim Lim, ${ }^{1}$ Tao Sun, ${ }^{2}$ and Naznin Sultana ${ }^{1,3}$ \\ ${ }^{1}$ Department of Clinical Sciences, Faculty of Biosciences and Medical Engineering, Universiti Teknologi Malaysia (UTM), \\ Skudai, 81310 Johor, Malaysia \\ ${ }^{2}$ Miniaturized Medical Devices Program, Institute of Microelectronics, Agency for Science, \\ Technology and Research (A ${ }^{* S T A R}$ ), Singapore \\ ${ }^{3}$ Advanced Membrane Technology Research Center, Universiti Teknologi Malaysia (UTM), Skudai, 81310 Johor, Malaysia \\ Correspondence should be addressed to Naznin Sultana; naznin@biomedical.utm.my
}

Received 17 September 2015; Accepted 22 November 2015

Academic Editor: Shafiul Chowdhury

Copyright (c) 2015 Mim Mim Lim et al. This is an open access article distributed under the Creative Commons Attribution License, which permits unrestricted use, distribution, and reproduction in any medium, provided the original work is properly cited.

\begin{abstract}
The fabrication of biocompatible and biodegradable scaffolds which mimic the native extracellular matrix of tissues to promote cell adhesion and growth is emphasized recently. Many polymers have been utilized in scaffold fabrication, but there is still a need to fabricate hydrophilic nanosized fibrous scaffolds with an appropriate degradation rate for skin tissue engineering applications. In this study, nanofibrous scaffolds of a biodegradable synthetic polymer, polycaprolactone (PCL), and blends of PCL with a natural polymer, gelatine $(\mathrm{Ge})$, in three different compositions: $85: 15,70: 30$, and $50: 50$ were fabricated via an electrospinning technique. The nanofibrous scaffold prepared from $14 \% \mathrm{w} / \mathrm{v}$ PCL/Ge $(70: 30)$ exhibited more balanced properties of homogeneous nanofibres with an average fibre diameter of $155.60 \pm 41.13 \mathrm{~nm}, 83 \%$ porosity, and surface roughness of $176.27 \pm 2.53 \mathrm{~nm}$. In vitro cell culture study using human skin fibroblasts (HSF) demonstrated improved cell attachment with a flattened morphology on the PCL/Ge $(70: 30)$ nanofibrous scaffold and accelerated proliferation on day 3 compared to the PCL nanofibrous scaffold. These results show that the PCL/Ge $(70: 30)$ nanofibrous scaffold was more favourable and has the potential to be a promising scaffold for skin tissue engineering applications.
\end{abstract}

\section{Introduction}

Research on developing biodegradable, biofunctional, and biocompatible nanosized materials has gained interest recently [1]. The nanometre range is the scale of proteins and other biological macromolecules and the scale at which functional interactions between these molecules take place inside and outside of cells. Hence, researchers are trying to fabricate nanosized biomaterial scaffolds which mimic living matter for various purposes, including tissue engineering and wound dressing applications. The development of nanosized scaffolds that mimic the architecture of skin tissue is one of the challenges in skin tissue engineering. There are many techniques in fabricating scaffolds. Among the various techniques of scaffold fabrication, the electrospinning technique is one of the most widely used techniques [2] to fabricate highly porous fibrous scaffolds on the micro- and nanoscales [3]. This is a nonmechanical processing strategy that is versatile and inexpensive. Fibrous scaffolds mimic the extracellular matrix (ECM) of the skin which functions as a structural support for cells and is favourable for cell attachment, proliferation, and differentiation. Studies have shown that fibrous scaffolds have greater advantages over nonfibrous scaffolds [4].

Electrospinning uses a high voltage electric field to create a charged jet of a polymer solution which is deposited on a grounded collector after travelling through the air. When high voltage is applied to the polymer solution, an electrostatic force will be formed and balanced with surface tension to form a cone at the tip of the needle, called a Taylor 
cone. By increasing the electric field, the polymer solution forms a fibre jet when the electrostatic force is greater than the surface tension. During ejection and elongation of the fibre jet, the solvent is evaporated, leaving only fibres which will be attracted to the grounded collector [5].

To fabricate fibrous scaffolds with the desired properties for skin tissue engineering, one of the most effective methods is polymer blending, especially blending of natural and synthetic polymers. Polycaprolactone (PCL) is a hydrophobic synthetic polymer which produces electrospun fibres that mimic the ECM but causes a reduction in cell contact and has a slow degradation rate $[6,7]$. Gelatine $(\mathrm{Ge})$ is a highly hydrophilic natural polymer which can be obtained easily and at low cost. The advantages of gelatine over PCL are that Ge is hydrophilic and does not cause a foreign body response [8]. Hence, blending of PCL and Ge will provide intermediate properties which will enhance hydrophilicity and be more favourable to cells.

Previously we reported the fabrication, the antibiotic drug loading, and the antibacterial properties of PCL/Ge scaffolds [9]. In this study, we report more elaborately about PCL and PCL/Ge nanofibrous scaffolds of different weight ratios produced using the electrospinning technique and their biological evaluation in vitro through cell culture. Formic acid was used as the solvent to dissolve PCL and Ge as formic acid is a less toxic volatile solvent that can be used to solidify fibres and reduce the formation of beads. Characterization was performed and compared among PCL and PCL/Ge nanofibrous scaffolds to determine the best scaffold for skin tissue engineering applications. Both scaffolds were tested for in vitro cell cytotoxicity and cell behaviour using human skin fibroblasts (HSF).

\section{Materials and Methods}

2.1. Materials. PCL (molecular weight 70,000-90,000), Ge powder (type A; porcine skin; $300 \mathrm{~g}$ Bloom), formic acid ( $\geq 95 \%$ ), phosphate buffered saline (PBS, P-3813, pH 7.4), fetal bovine serum (FBS, F7524), and glutaraldehyde (Grade I, 50\% in $\mathrm{H}_{2} \mathrm{O}$ ) were purchased from Sigma-Aldrich. Silver nitrate $\left(\mathrm{AgNO}_{3}\right.$, Grade $\mathrm{AR}, 169,87 \mathrm{~g} / \mathrm{mol}$ ), ethanol (approximate $96 \%$, Grade AR), and dimethyl sulfoxide (DMSO, Grade AR) were supplied from QRec. 3-(4,5-Dimethylthiazol-2-yl)-2,5diphenyltetrazolium bromide (MTT, M6494) was purchased from Molecular Probes by Life Technologies and Dulbecco's Modified Eagle Medium (DMEM, high glucose, 12100-038), trypan blue stain $(0.4 \%, 15250-061)$, trypsin $(0.25 \%, 1 \mathrm{x}$, 25200-056), and penicillin-streptomycin (Pen Strep, 15140122) were supplied from Gibco by Life Technologies. HSF (1184) were purchased from American Type Culture Collection (ATCC).

\subsection{Fabrication and Characterization of PCL and PCL/Ge Fibrous Scaffolds}

2.2.1. Preparation of PCL and PCL/Ge Solution. The PCL solution and the PCL/Ge solution with different weight ratios $(85: 15,70: 30$, and $50: 50)$ were prepared by dissolving the polymers in formic acid. Solutions were stirred using a magnetic stirrer for 3 hours at room temperature until all polymers were dissolved.

2.2.2. Electrospinning of PCL and PCL/Ge Fibrous Scaffolds. A NaBond Nanofibre Electrospinning Unit (China) was used for the electrospinning process. The as-prepared PCL and PCL/Ge solutions were loaded into a $10 \mathrm{~mL}$ syringe. A 23 gauge stainless steel needle was attached to the syringe as a nozzle and the syringe was placed on a syringe pump (NE300, New Era Pump Systems, Inc.). A piece of aluminium foil was placed at the collector for the deposition of fibres. An emitting electrode with a positive charge was attached to the nozzle and the grounding electrode was attached to the collector. A high voltage of $15 \mathrm{kV}$ was applied for 2 hours with a $10 \mathrm{~cm}$ distance between the capillary tip and the collector.

2.2.3. Characterization. All nanofibrous scaffolds were sputter-coated with gold. The morphology of the coated scaffolds was viewed under a field emission scanning electron microscope (FESEM, SU8020, Hitachi). Fibre diameters were measured using Image J software and average fibre diameters were calculated by taking at least 20 measurements. All fibre diameters are presented as mean \pm standard deviation (SD).

The porosity and density of the nanofibrous scaffolds were measured by the liquid displacement method. Ethanol was used as the displacement liquid. The initial weight $(w)$ of the scaffolds was noted and immersed in a known volume $\left(V_{1}\right)$ of ethanol for 5 minutes. The volume of ethanol in the ethanol-impregnated fibre was recorded as $V_{2}$. The residual volume after the ethanol-impregnated fibre was removed was recorded as $V_{3}$. The total volume, density, and porosity of the fibres were calculated using the equations below. This experiment was repeated three times and the average value was calculated.

Total volume of fibre was

$$
V=V_{2}-V_{3}
$$

Density $(d)$ of the fibre was

$$
d=\frac{w}{V_{2}-V_{3}}
$$

Porosity $(\varepsilon)$ of the fibre was

$$
\varepsilon=\frac{\left(V_{1}-V_{3}\right)}{\left(V_{2}-V_{3}\right)} .
$$

The surface topography and surface roughness of the nanofibrous scaffolds were assessed by atomic force microscopy (AFM) (TT-AFM, AFM Workshop) using a preset scanning area of 15 microns by 15 microns and a scan rate of $0.3002 \mathrm{~Hz}$. Data are the arithmetic mean roughness (average roughness), and $R_{a}$ was calculated by taking an average of three values of $R_{a}$. 


\subsection{In Vitro HSF Cell Cytotoxicity and Behaviour on PCL and PCL/Ge Nanofibrous Scaffolds}

2.3.1. Preparation and Sterilization of Nanofibrous Scaffolds. PCL and PCL/Ge $(70: 30)$ nanofibrous scaffolds were cut into circular discs $(1.6 \mathrm{~cm}$ in diameter) and washed three times using PBS with $1 \%$ Pen Strep followed by UV radiation exposure for 2 hours.

2.3.2. Optimization of Cell Number on 24-Well Plate. The number of cells seeded on the nanofibrous scaffolds in 24well plates was determined by optimizing the number of cells. Thus, $500,1 \times 10^{3}, 5 \times 10^{3}, 10 \times 10^{3}, 20 \times 10^{3}$, and $50 \times 10^{3}$ HSF cells/well were seeded in 24-well plates and cultured in DMEM containing 10\% FBS and 1\% Pen Strep in a carbon dioxide $\left(\mathrm{CO}_{2}\right)$ incubator (INC153med, Memmert) $\left(37^{\circ} \mathrm{C}, 5 \%\right.$ $\mathrm{CO}_{2}$ ) for 7 days. DMEM was changed every two days. After day 7, the HSF distribution and confluence were viewed under an inverted microscope (Nikon) and the suitable number of cells for 7 days of culture was determined.

2.3.3. Cell Culture with HSF Cells. HSF cells were used to investigate cell behaviour on both the PCL and PCL/Ge $(70: 30)$ nanofibrous scaffolds. HSF cells were cultured in DMEM containing $10 \% \mathrm{FBS}$ and $1 \%$ Pen Strep in $\mathrm{CO}_{2}$ incubator $\left(37^{\circ} \mathrm{C}, 5 \% \mathrm{CO}_{2}\right)$. DMEM was replaced every two days. When HSF cells reached $80 \%$ confluence, they were trypsinized and counted using a haemocytometer (Counting Chambers, Neubauer-Improved, Hirschmann). A density of $10 \times 10^{3}$ cells/well was seeded on each scaffold in 24 -well plates for cell counting, morphology, and cell cytotoxicity tests at 4 hours and 1,3 , and 7 days.

2.3.4. Cell Cytotoxicity Using the MTT Assay. The MTT assay was used to test for cell cytotoxicity on the PCL and PCL/Ge $(70: 30)$ nanofibrous scaffolds. Cells cultured on wells without a scaffold were used as the control. The mechanism of this assay is based on metabolically active cells reducing the tetrazolium salt of MTT to purple formazan. $10 \times 10^{3}$ cells/well were seeded on the scaffolds for three days in 24-well plates. This experiment was performed in triplicate. After three days, DMEM was removed. New DMEM with the MTT solution $(5 \mathrm{~g} / \mathrm{L})$ was added to each well followed by incubation for 4 hours. The plates were wrapped with aluminium foil as MTT is sensitive to light. After 4 hours, purple formazan had formed. Medium was removed and added with $1 \mathrm{~mL}$ DMSO to dissolve the formazan. After all the formazan crystals were dissolved, $100 \mu \mathrm{L}$ aliquots was transferred to a 96-well plate, with five replicates per sample. DMSO was set as the blank. Absorbance was measured at a wavelength of $570 \mathrm{~nm}$ using a microplate spectrophotometer (Epoch, BioTek).

2.3.5. Cell Counting Using Dye Exclusion Method (Trypan Blue). Cell attachment was evaluated by cell counting at 24 hours, 1 day, and 3 days. The dye exclusion method using trypan blue was used. After each time point, the scaffolds were moved to another well, washed with PBS, and trypsinized for 3 minutes. Then, $500 \mu \mathrm{L}$ DMEM was added to stop the trypsinization process. The cell suspension was centrifuged and DMEM was added to the cell pellet followed by trypan blue. Dead cells were stained blue. The number of viable and dead cells was counted using a haemocytometer under a microscope. The number of cells per $\mathrm{mL}(N)$ was calculated using the formula below, where $N$ is the number of viable cells. Cell counting was done in triplicate:

$$
N=\frac{n}{4} \times 10,000 \times \text { dilution factor. }
$$

2.3.6. Morphological Characterization of Cultured Cells. HSF cell morphology on the PCL and PCL/Ge (70:30) nanofibrous scaffolds was observed after 4 hours, 1 day, 3 days, and 7 days by using a scanning electron microscope (SEM, TM3000 Tabletop, Hitachi). After each time point, scaffolds were moved to another well, washed with PBS, and fixed with $4 \%$ glutaraldehyde for 1 hour at $4^{\circ} \mathrm{C}$. After 1 hour, scaffolds underwent gradual dehydration in 30\%, 50\%, 70\%, 95\%, and $100 \%$ ethanol and were air-dried in a biosafety cabinet (BSC) (1300 Series Class II, Type A2, Thermo Scientific). The dried samples were sputter-coated with platinum for 20 seconds and observed under SEM.

2.4. Statistical Analysis. All data are expressed as mean values \pm SD. Statistical analyses were carried out using ANOVA. A $p$ value $<0.05$ was considered statistically significant and a $p$ value $<0.01$ was extraordinarily significant. ${ }^{*} p<0.05 ;{ }^{* *} p<$ $0.01 ;$ and $^{* * *} p<0.001$.

\section{Results and Discussion}

3.1. Fabrication and Characterization of PCL and PCL/Ge Nanofibrous Scaffolds. Previous study by our research group found that a $14 \% \mathrm{w} / \mathrm{v} \mathrm{PCL} / \mathrm{Ge}$ solution gave better results in terms of fibre morphology [9]. In this study, a $14 \% \mathrm{w} / \mathrm{v}$ $\mathrm{PCL} / \mathrm{Ge}$ solution was used with different weight ratios; that is, $85: 15,70: 30$, and $50: 50 \mathrm{PCL}$ and Ge blends were investigated. The $14 \% \mathrm{w} / \mathrm{v}$ PCL solution was also used to fabricate PCL nanofibrous scaffolds and compared with $14 \% \mathrm{w} / \mathrm{v}$ PCL/Ge nanofibrous scaffolds. The electric field, flow rate, and distance between the capillary tip and the collector were kept constant at $15 \mathrm{kV}, 1 \mathrm{~mL} / \mathrm{h}$, and $10 \mathrm{~cm}$ for every preparation. Formic acid was used as the solvent to dissolve PCL and PCL/Ge.

PCL is soluble in organic solvents [10], while Ge is soluble in aqueous solutions of polyhydric alcohols and highly polar organic solvents (acetic acid, trifluoroethanol, formamide, and formic acid). Ge is insoluble in less polar organic solvents including primary alcohols and chloroform [11].

Formic acid had been reported as a good solvent to dissolve Ge [12] and PCL [13] individually. Since Ge is insoluble in chloroform, formic acid was chosen to dissolve PCL and Ge for PCL/Ge fibrous scaffold fabrication. Characterization of the $14 \% \mathrm{w} / \mathrm{v}$ PCL and $14 \% \mathrm{w} / \mathrm{v}$ PCL/Ge $(85: 15$, $70: 30$, and $50: 50)$ nanofibrous scaffolds fabricated using formic acid as the solvent was performed to compare the scaffolds in terms of morphology, average fibre diameter, pore 
TABLE 1: Processing parameters and average fiber diameter of PCL and PCL/Ge nanofibrous scaffolds. Solution concentration, electric field, flow rate, and distance between tip and collector were kept constant at $14 \% \mathrm{w} / \mathrm{v}, 15 \mathrm{kV}, 1 \mathrm{~mL} / \mathrm{h}$, and $10 \mathrm{~cm}$. All diameters are presented as mean $\pm \mathrm{SD}(n=30)$.

\begin{tabular}{lcccc}
\hline Polymer & Weight ratio of PCL: Ge & Temperature $\left({ }^{\circ} \mathrm{C}\right)$ & Humidity $(\%)$ & Average fiber diameter $(\mathrm{nm})$ \\
\hline PCL & - & 21.9 & 50 & $88.02 \pm 27.83$ \\
PCL/Ge & $85: 15$ & 21.4 & 52 & $143.39 \pm 28.60$ \\
PCL/Ge & $70: 30$ & 23.1 & 50 & $155.60 \pm 41.13$ \\
PCL/Ge & $50: 50$ & 23.1 & 50 & $219.22 \pm 87.93$ \\
\hline
\end{tabular}

size, wettability, porosity, density, chemical bonding, surface topography, surface roughness, and in vitro degradation rate.

3.1.1. Morphology. Figure 1 shows the morphological images taken by FESEM and the fibre diameter distribution of the PCL and PCL/Ge nanofibrous scaffolds. The average fibre diameters of the PCL and PCL/Ge nanofibrous scaffolds are shown in Table 1 . Both the PCL and PCL/Ge fibrous scaffolds were in the nanometre range. A nanosized fibre diameter gives a higher surface area to volume ratio and provides a suitable environment for cells during cell attachment and proliferation.

It has been reported that electrospinning of PCL in formic acid results in a structure mainly composed of droplets using a $13 \% \mathrm{w} / \mathrm{v}$ PCL solution at $15 \mathrm{kV}, 2 \mathrm{~mL} / \mathrm{h}$, and a $6 \mathrm{~cm}$ distance at $30 \%$ humidity [13]. However, in this study, nanosized homogeneous fibres were successfully fabricated using a $14 \% \mathrm{w} / \mathrm{v}$ PCL solution at $15 \mathrm{kV}, 1 \mathrm{~mL} / \mathrm{h}$, and a $10 \mathrm{~cm}$ distance at $50 \pm 2 \%$ humidity. It is essential for biomedical scaffolds to have a nanosized structure which mimics the natural ECM morphology to promote optimal cell growth.

From these results, it was found that the addition of $\mathrm{Ge}$ increased the fibre diameter in the PCL/Ge nanofibrous scaffolds. With increasing Ge content, the average fibre diameter increased. As compared to other results, these findings are contradictory. It has been reported that, with the addition of $\mathrm{Ge}$, the fibre diameter decreased and the average fibre diameter was inversely proportional to the concentration of Ge [14]. However, 70\% of the dry dermis weight in ECM is collagen fibres with a fibre diameter in the range of 100$500 \mathrm{~nm}[15]$. In this study, PCL/Ge $(85: 15,70: 30$, and $50: 50)$ nanofibrous scaffolds were within this range and it was concluded that these nanofibrous scaffolds would provide a similar environment for the growth of fibroblasts in the dermis layer.

3.1.2. Porosity and Density. Table 2 shows the porosity and density measurement results of the PCL and PCL/Ge nanofibrous scaffolds with a composition $70: 30$. The PCL nanofibrous scaffold had a porosity of $85 \%$ and a density of $0.011 \mathrm{~g} / \mathrm{cm}^{3}$ while the PCL/Ge $(70: 30)$ nanofibrous scaffold had a porosity of $83 \%$ and a density of $0.012 \mathrm{~g} / \mathrm{cm}^{3}$. Based on these results, porosity is indirectly proportional to density.

Porosity and density are key metrics of tissue engineering scaffolds and can be measured by the liquid displacement method. Porosity is the percentage of the void (empty space) volume of the fibre and is different from pore size which is
TABLE 2: Porosity and density measurement of PCL and PCL/Ge $(70: 30)$ nanofibrous scaffolds $(n=3)$.

\begin{tabular}{lcc}
\hline Nanofibrous scaffolds & Porosity $(\%)$ & Density $\left(\mathrm{g} / \mathrm{cm}^{3}\right)$ \\
\hline PCL & $85 \pm 2.5$ & $0.011 \pm 0.005$ \\
PCL/Ge $70: 30$ & $83 \pm 1.8$ & $0.012 \pm 0.004$ \\
\hline
\end{tabular}

the measurement of interfibre size. In this study, the liquid displacement method was conducted using ethanol. Water was not used due to the hydrophobic properties of PCL and as Ge can be dissolved in water. Ethanol is a suitable displacement liquid as PCL and Ge are insoluble in ethanol. Ethanol can penetrate easily into the pores and will not lead to shrinkage or swelling of the fibres. The porosity results of a PCL/Ge (70:30) scaffold fabricated using tetrafluoroethylene was around $60-70 \%$ [16]. In our study, the PCL/Ge $(70: 30)$ scaffold fabricated using formic acid showed much higher porosity, that is, $83 \%$. This shows that the solvent plays an important role in influencing the porosity of the fibres as evaporation of the solvent during the electrospinning process leads to the formation of pores.

The preferred porosity for cell penetration is within the range of $60-90 \%$ [17]. Both the PCL and PCL/Ge $(70: 30)$ nanofibrous scaffolds had porosity within that range, which indicates that both scaffolds are suitable for cell penetration. In previous studies using cell culture, Ge was found to dissolve slowly to create spaces for cell migration and PCL/Ge showed good elongation as well as deformation properties which provides space for cellular penetration [18]. Porosity is an important parameter for tissue engineering scaffolds. For skin regeneration applications, nanofibrous scaffolds should be highly porous.

3.1.3. Surface Topography and Surface Roughness. AFM is designed primarily to assess the surface topography of a substance in contact mode, noncontact mode, or tapping mode. In this study, contact mode was used where the surface topography is obtained by "dragging" the tip along the scaffold surface. The detailed morphology of PCL and PCL/Ge (70:30) nanofibrous scaffolds in two dimensions (2D) and three dimensions (3D) were obtained and are shown in Figure 2. These images show a 2D and 3D structure with a random fibre orientation.

$R_{a}$ of PCL and PCL/Ge $(70: 30)$ nanofibrous scaffolds was $131.56 \pm 17.55 \mathrm{~nm}$ and $176.27 \pm 2.53 \mathrm{~nm}$, respectively. Incorporation of $\mathrm{Ge}$ increased the surface roughness. It was discovered that the surface roughness of the PCL nanofibrous 

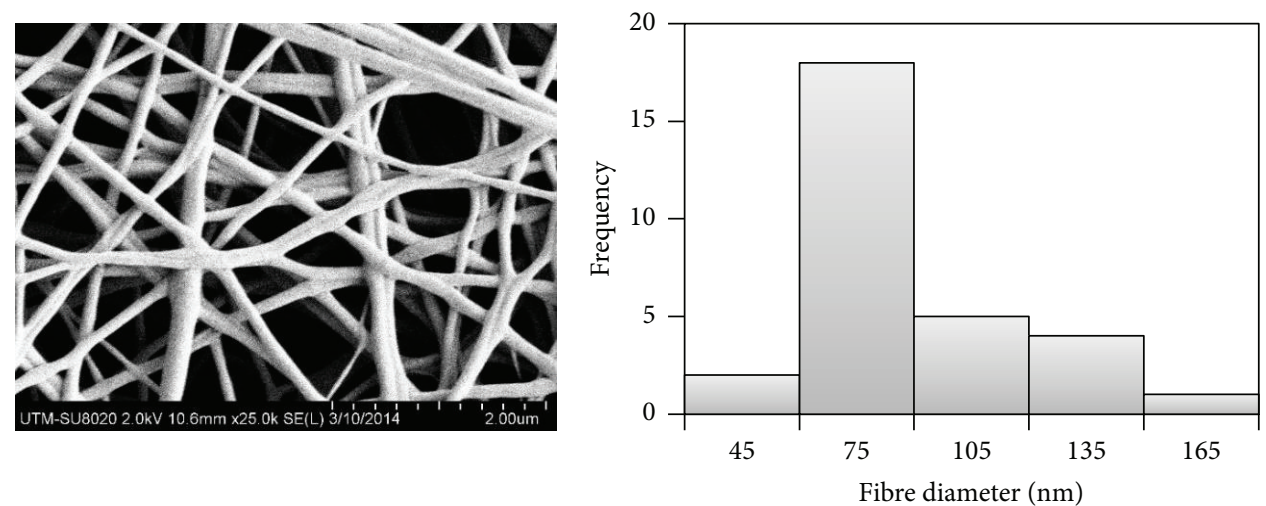

(a)
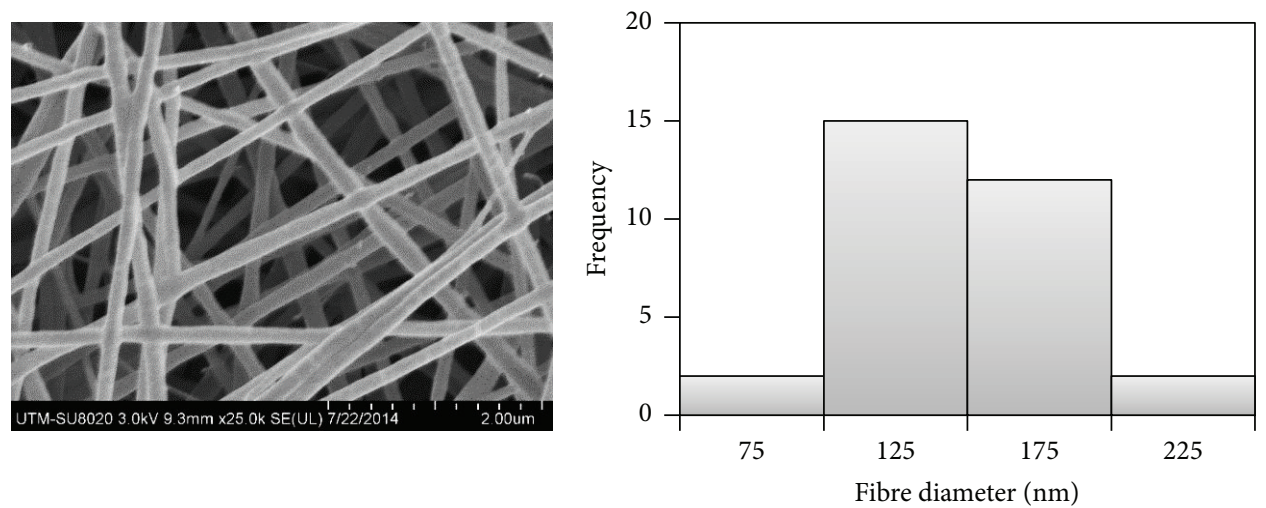

(b)
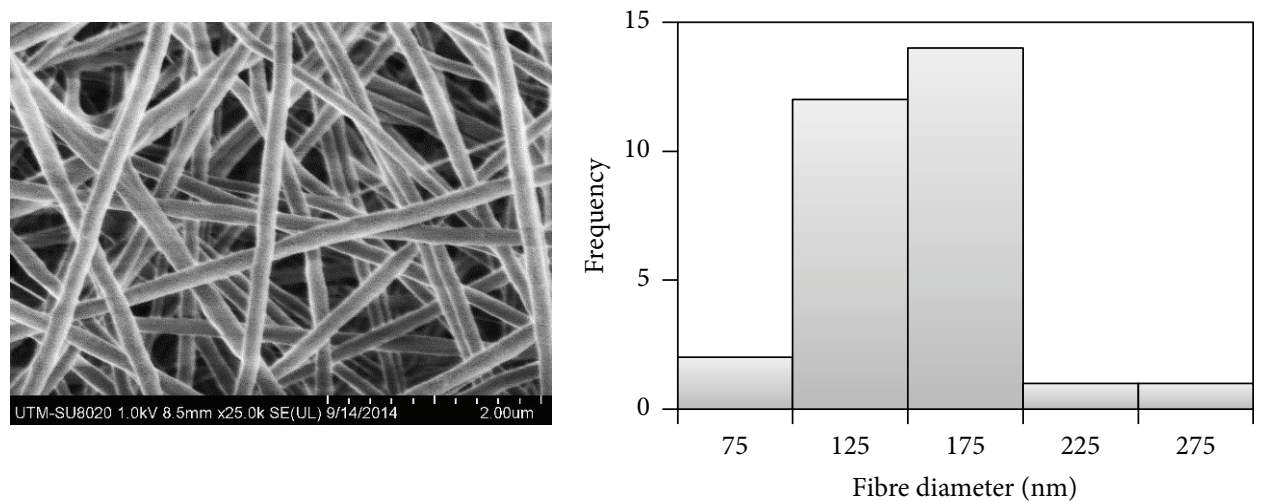

(c)
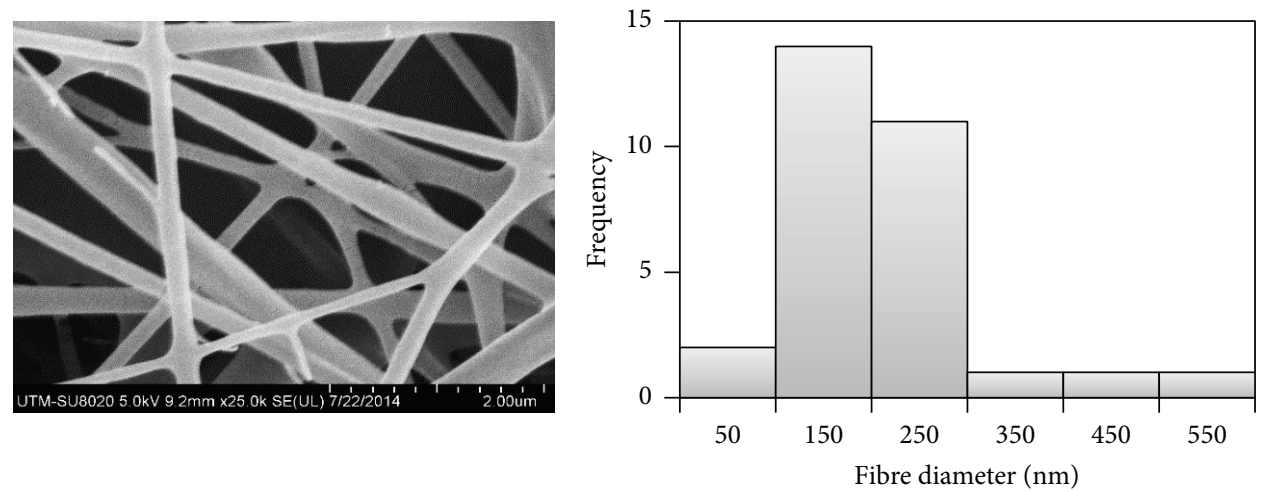

(d)

FIGURE 1: FESEM images (left) and fibre diameter distribution (right) of nanofibrous scaffolds fabricated using formic acid at a concentration of 14\% w/v: 25000x magnification (a) PCL, (b) PCL/Ge (85:15), (c) PCL/Ge (70:30), and (d) PCL/Ge (50:50). 

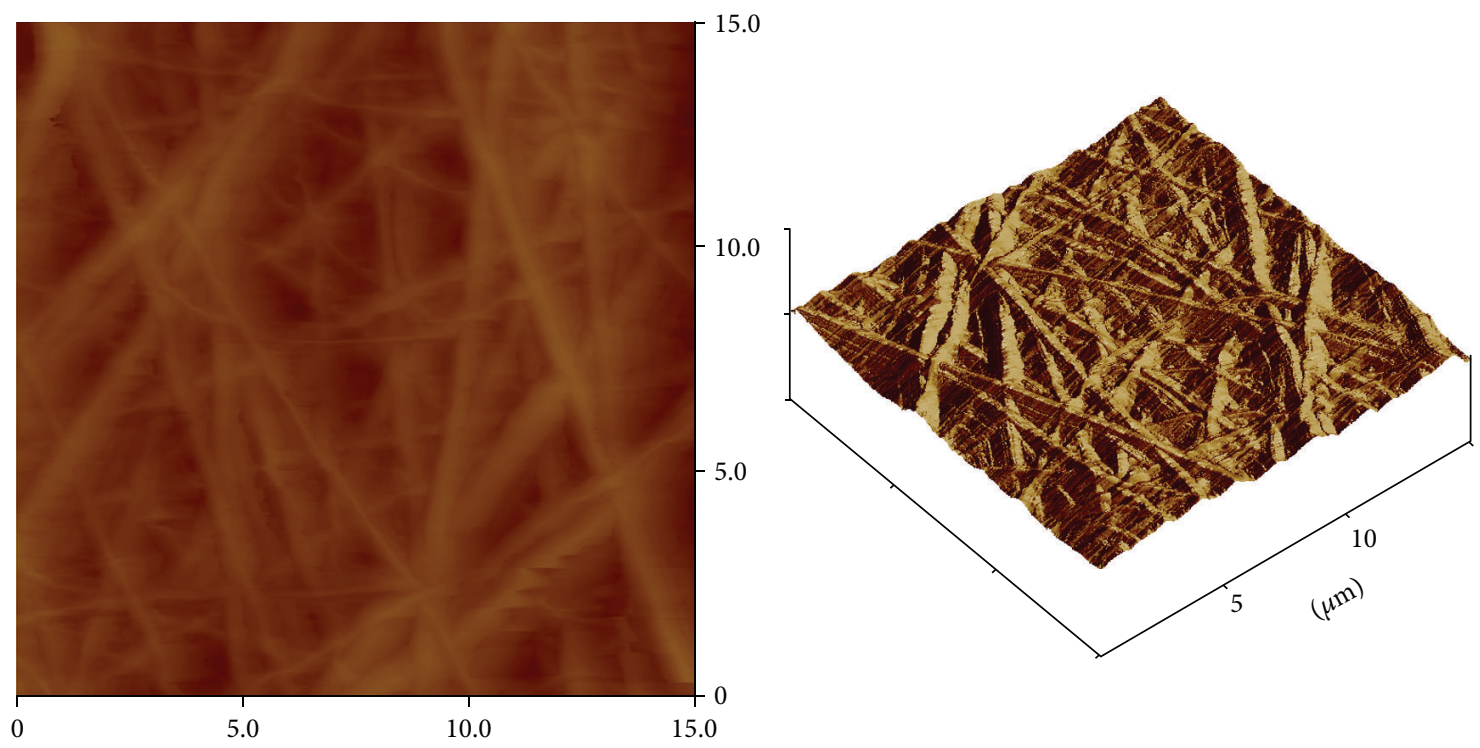

(a)

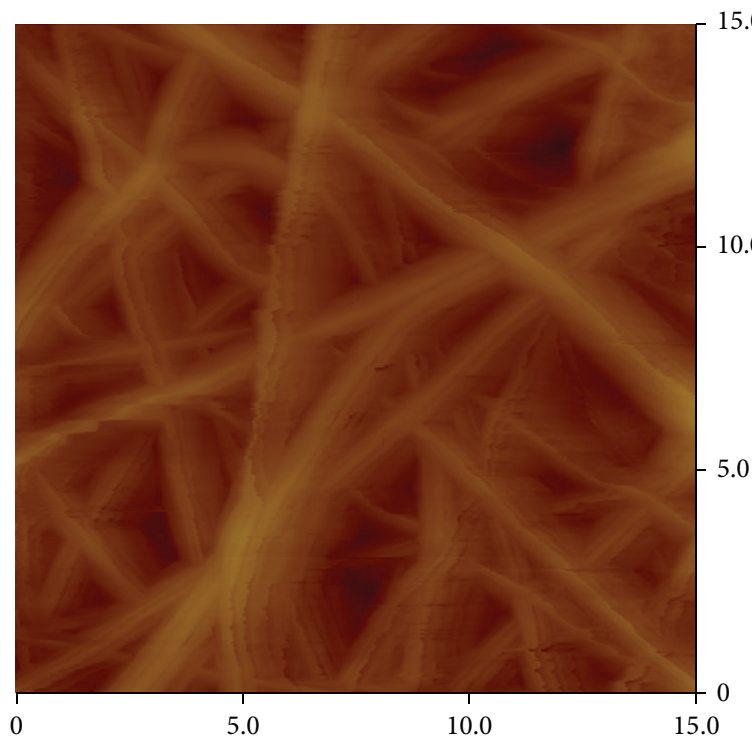

15.0

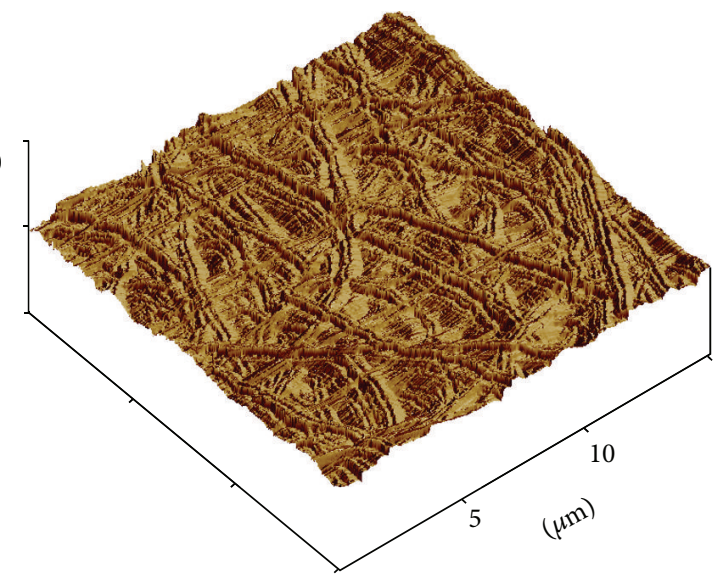

(b)

Figure 2: AFM images of nanofibrous scaffolds in 2D images (left) and 3D images (right): (a) PCL and (b) PCL/Ge (70:30).

scaffold was less than that of the PCL/Ge $(70: 30)$ nanofibrous scaffold. Previous studies have shown that increasing the fibre diameter will lead to an increase in surface roughness [19]. This is similar to our study where the PCL nanofibrous scaffold had a smaller fibre diameter with a lower surface roughness, but the PCL/Ge nanofibrous scaffold had a larger fibre diameter with a higher surface roughness.

\subsection{In Vitro HSF Cell Cytotoxicity and Behaviour on PCL and PCL/Ge (70:30) Nanofibrous Scaffolds}

3.2.1. Optimization of Cell Number on 24-Well Plates. Variable cell numbers, that is, $500,1 \times 10^{3}, 5 \times 10^{3}, 10 \times 10^{3}$, $20 \times 10^{3}$, and $50 \times 10^{3}$ cells/well, were cultured on 24 -well plates. On day 7 , the percentage of confluence for $500,1 \times 10^{3}$, $5 \times 10^{3}$, and $10 \times 10^{3}$ cells/well was estimated to be $30 \%$, $50 \%, 70 \%$, and $90 \%$, respectively. For $20 \times 10^{3}$ and $50 \times 10^{3}$ cells/well, cells were overconfluent and had started to detach. From these data, $10 \times 10^{3}$ cells/well were selected as the optimum number of cells seeded in 24-well plates in our study as this number of cells reached confluence at day 7 .

Optimization of cell number is an important step in cell culture to determine the concentration of cells to be seeded in a known volume of culture medium before culturing. Skin cells (HSF) were cultured in this study as the main purpose of our research was to study skin tissue engineering applications. The duration of our cell culture work was 7 days. Hence, a suitable number of cells had to be determined 


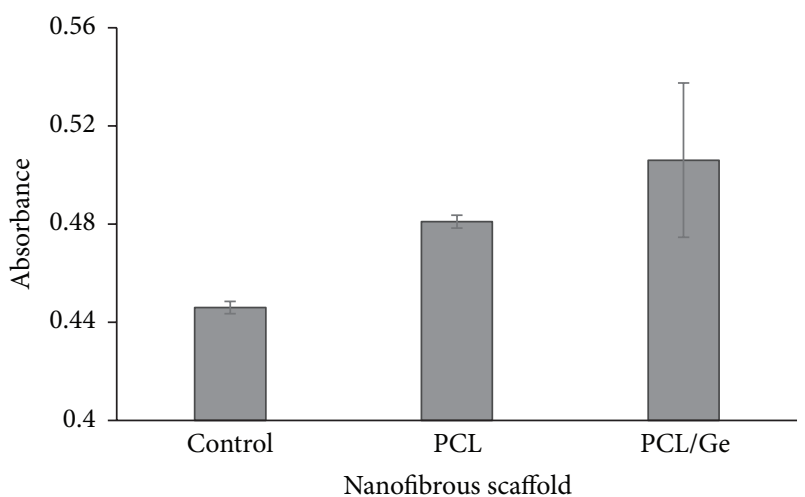

FIgURE 3: Cell cytotoxicity on control, PCL, and PCL/Ge (70:30) nanofibrous scaffolds. Data are representative of three independent experiments and are plotted as mean $\pm \mathrm{SD}(n=3) ;{ }^{*} p<0.05$.

to make sure that there was optimum cell growth and not overconfluence during the 7 days of culture.

3.2.2. Cell Cytotoxicity Using the MTT Assay. PCL and PCL/Ge $(70: 30)$ nanofibrous scaffolds were tested for cell cytotoxicity on day 3 using a quantitative method, the MTT assay. In this study, HSF cells cultured on the plate without any scaffold were used as the control. The cytotoxicity results are shown in Figure 3.

Both PCL and PCL/Ge $(70: 30)$ nanofibrous scaffolds gave high absorbance and the absorbance was higher than the control. The absorbance of the PCL/Ge $(70: 30)$ nanofibrous scaffold was slightly higher than that of the PCL nanofibrous scaffold.

This showed that both of these scaffolds were nontoxic to cells. This result is supported as PCL and Ge have already been approved by the FDA as being safe and there is no evidence to demonstrate a hazard to humans [20]. Cells grew and proliferated well on both scaffolds. Higher absorbance than the control showed that the nanofibrous scaffolds enhanced the growth of cells as compared to the surface of the plate. Both of these scaffolds provided favourable substrates for cells as the fibrous scaffolds mimicked the ECM and provided a high surface area to volume ratio for cell attachment and proliferation. The absorbance of the PCL/Ge $(70: 30)$ nanofibrous scaffold was slightly higher than the PCL nanofibrous scaffold due to the hydrophilicity of the PCL/Ge $(70: 30)$ nanofibrous scaffold. Cells are more likely to grow on a hydrophilic surface compared to a hydrophobic surface. Furthermore, there is a biochemical interaction between cells and collagen. This interaction is the binding of collagen I to receptors on the cell membrane which is mediated by the ECM glycoprotein fibronectin [21]. Ge is derived from collagen by hydrolysis and its structure is similar to that of collagen. Therefore, it can be concluded that blending of Ge with PCL enhanced the interaction between cells and the PCL/Ge $(70: 30)$ nanofibrous scaffold.

3.2.3. Cell Counting Using the Dye Exclusion Method (Trypan Blue). HSF cells were seeded on PCL and PCL/Ge $(70: 30)$ scaffolds for 4,24 , and 72 hours. The number of cells was

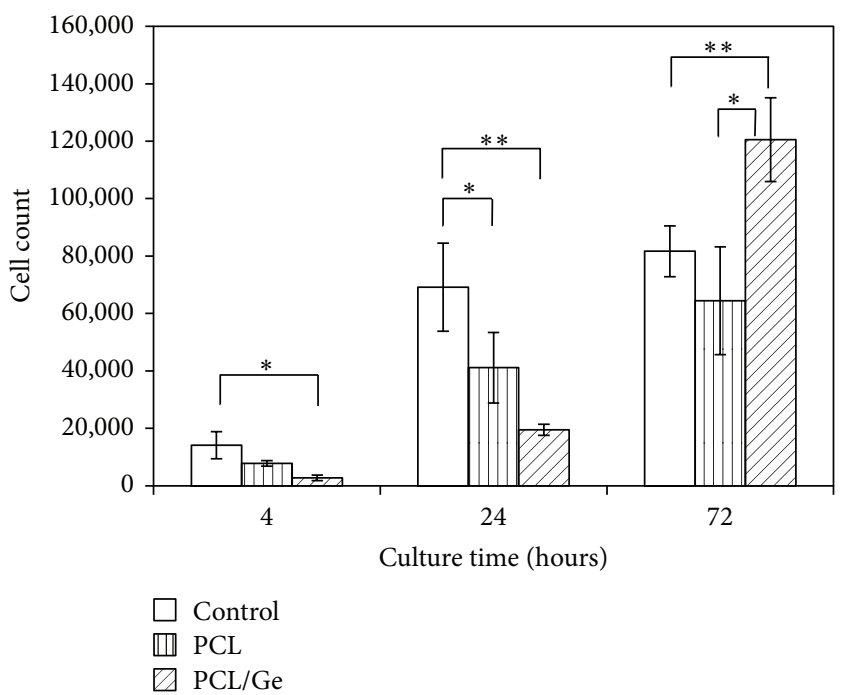

FIGURE 4: Cell counting results of control, PCL, and PCL/Ge $(70: 30)$ nanofibrous scaffolds at 4, 24, and 72 hours using the dye exclusion method (trypan blue). Data are representative of three independent experiments and are plotted as mean $\pm \mathrm{SD}(n=3) ;{ }^{*} p<0.05,{ }^{* *} p<$ 0.01 .

counted by the dye exclusion method using trypan blue in three replicates and the average value was calculated. Trypan blue is a dye that can differentiate between living and dead cells. It can selectively colour dead cells blue and living cells will be bright and refractile. Figure 4 shows the results of the viable cell count. Wells with only cells were used as the control. In this study, no dead cells were observed, but the growth of cells on each sample was different. The number of cells on the PCL and PCL/Ge (70:30) nanofibrous scaffolds increased over time. However, the cell number on the PCL nanofibrous scaffold was less than that in the control. For the PCL/Ge (70:30) nanofibrous scaffold, the cell number was lower at 4 and 24 hours but then increased rapidly and significantly by 72 hours. All of the data are statistically significant.

The high porosity of both nanofibrous scaffolds provided an optimum growth environment for cells by giving more space for nutrient and metabolic waste exchange. For the PCL nanofibrous scaffold, the number of cells increased with time, but the cell number was less than that in the control, indicating that cells were favoured the plate surface over the PCL nanofibrous scaffold. Surface properties are an important factor that will affect cell growth. Cells prefer hydrophilic surfaces. A hydrophilic surface enhances the effective adhesion of cells and enhances cell migration. However, others have shown that hepatocytes attached and proliferated on PCL nanofibres [22]. Cells grew on the hydrophobic fibrous scaffold since the fibre structure mimics ECM, but they had a slower growth rate. For the hydrophilic PCL/Ge (70:30) nanofibrous scaffold, the cell number was lower at 24 hours but then increased rapidly and significantly by 72 hours. This shows that the PCL/Ge $(70: 30)$ nanofibrous scaffold accelerated the proliferation and differentiation of HSF cells. 


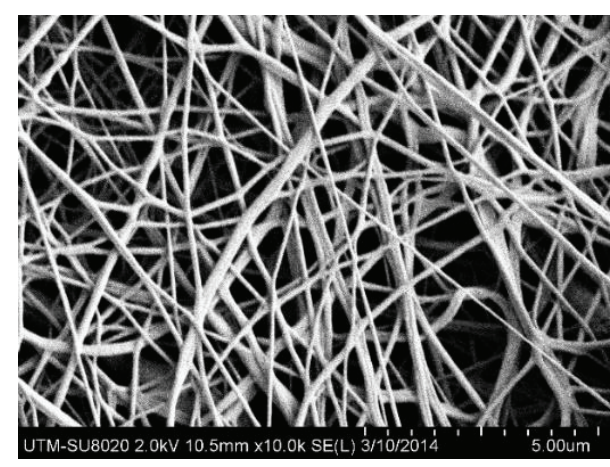

(a)

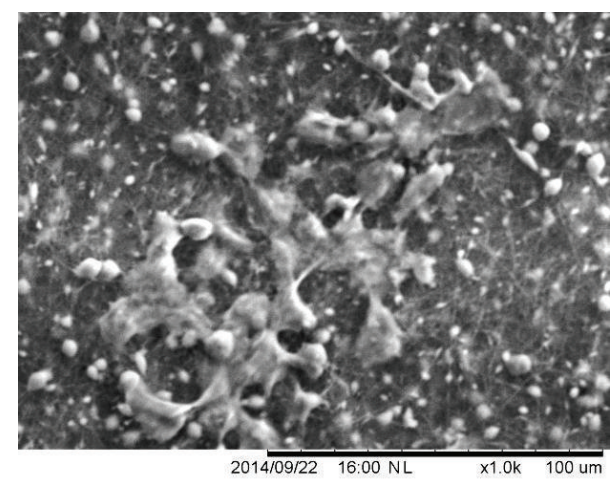

(c)

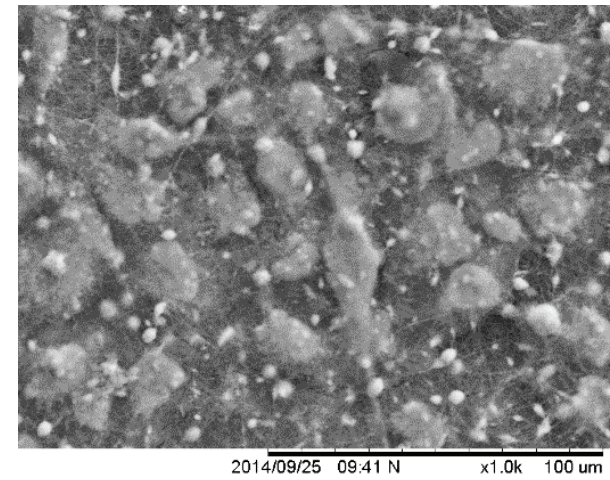

(e)

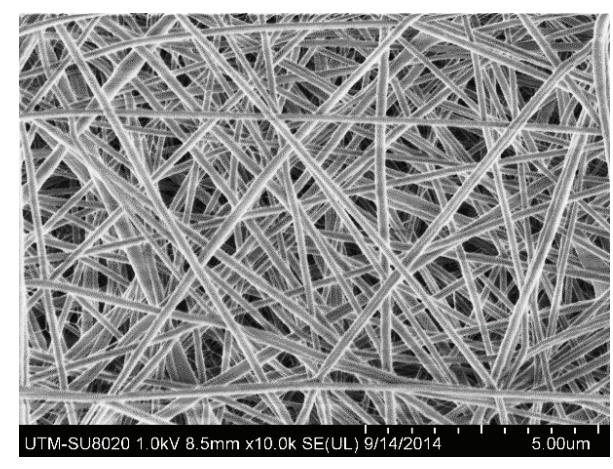

(b)

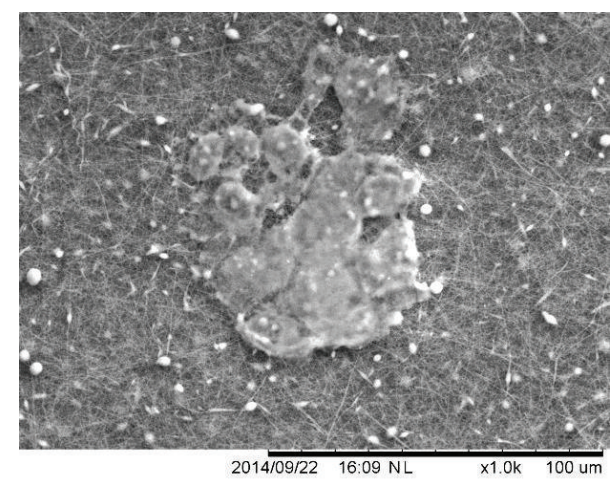

(d)

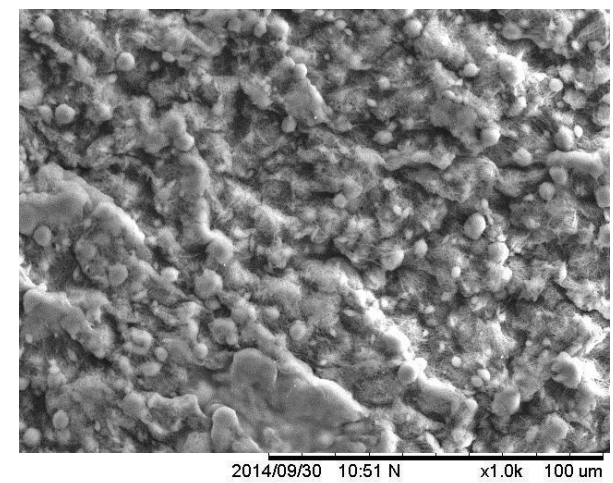

(f)

FIGURE 5: Morphology of HSF cultured on PCL and PCL/Ge nanofibrous scaffolds at different time points (a, b) before culturing; magnification 10000x, (c) 4 hours, (d) day 1, (e) day 3, and (f) day 7; magnification 1000x.

3.2.4. Morphological Characterization of Cultured Cells. The morphology of HSF cultured on the PCL and PCL/Ge $(70: 30)$ nanofibrous scaffolds was studied by SEM at 4 hours, 1 day, 3 days, and 7 days. These results are shown in Figure 5.

PCL and PCL/Ge $(70: 30)$ nanofibrous scaffolds were tested for cell cytotoxicity and the results show that both scaffolds were nontoxic to cells. Since both scaffolds were not harmful to cells, it was also found in the SEM images that cells were growing on the scaffolds. However, these cells were randomly orientated. With increasing culture time, cells were spread on the scaffolds and increased in size. At day 7, nearly $100 \%$ cell distribution was observed where the surface of the scaffold was almost completely covered by cells. Cells had grown into a confluent state, forming a monolayer.

On the PCL nanofibrous scaffold, cells aggregated and had a globular morphology with extended filopodia. However, on the PCL/Ge $(70: 30)$ nanofibrous scaffold, cells were more flattened, but some globular morphology could be seen in the SEM images taken at 4 hours and 1 day. In the SEM image at 3 days, cells no longer maintained a globular morphology but exhibited a flattened and stretched morphology with increased surface area. On day 7 , the population of cells had spread with a flattened morphology on the PCL/Ge $(70: 30)$ nanofibrous scaffold. 
Aggregation and the globular morphology of the cells on the PCL nanofibrous scaffold indicated poor attachment of the cells on the PCL scaffold. Aggregation of cells occurred due to the hydrophobicity of PCL. This result is similar to a previous study [23] showing that a hydrophobic surface will lead to poor cell attachment [24]. Although there was poor attachment of cells on the PCL nanofibrous scaffold, the number of cells was increased. Cells were still growing but with poor attachment. Slow attachment of cells was initially found on the PCL/Ge $(70: 30)$ nanofibrous scaffold. The flattened and stretched morphology with increased surface area of the cells on the PCL/Ge $(70: 30)$ nanofibrous scaffold at day 3 showed good cell to cell and cell to scaffold interactions.

\section{Conclusions}

Nanofibrous scaffolds of PCL and blends of PCL and Ge with weight ratios of $85: 15,70: 30$, and $50: 50$ were successfully fabricated using the electrospinning technique with formic acid as the solvent at a concentration of $14 \% \mathrm{w} / \mathrm{v}$. The $14 \% \mathrm{w} / \mathrm{v}$ PCL/Ge (70:30) nanofibrous scaffolds exhibited desirable properties, including homogeneous nanofibres with an average fibre diameter of $155.60 \pm 41.13 \mathrm{~nm}, 83 \%$ porosity. MTT assay showed that $14 \% \mathrm{w} / \mathrm{v}$ PCL/Ge $(70: 30)$ nanofibrous scaffolds were nontoxic to HSF cells. There were better cell to cell and cell to scaffold interactions on the PCL/Ge $(70: 30)$ nanofibrous scaffold compared to the PCL nanofibrous scaffold. This study demonstrated that the $14 \% \mathrm{w} / \mathrm{v} \mathrm{PCL} / \mathrm{Ge}$ $(70: 30)$ nanofibrous scaffold had the potential to be used for skin tissue engineering applications.

\section{Conflict of Interests}

The authors declare that there is no conflict of interests.

\section{Acknowledgments}

This work was supported by MOHE and GUP Tier 1 Grants (Vot: 06H84) and FRGS (Vot: 4F507). The authors would also like to acknowledge the lab facilities provided by Universiti Teknologi Malaysia (UTM) and the Agency of Science, Technology, and Research (A*STAR).

\section{References}

[1] T. Sun, T. H. Khan, and N. Sultana, "Fabrication and in vitro evaluation of nanosized hydroxyapatite/chitosan-based tissue engineering scaffolds," Journal of Nanomaterials, vol. 2014, Article ID 194680, 8 pages, 2014.

[2] M. I. Hassan, T. Sun, and N. Sultana, "Fabrication of nanohydroxyapatite/poly(caprolactone) composite microfibers using electrospinning technique for tissue engineering applications," Journal of Nanomaterials, vol. 2014, Article ID 209049, 7 pages, 2014.

[3] F. Roozbahani, N. Sultana, A. F. Ismail, and H. Nouparvar, "Effects of chitosan alkali pretreatment on the preparation of electrospun PCL/chitosan blend nanofibrous scaffolds for tissue engineering application," Journal of Nanomaterials, vol. 2013, Article ID 641502, 6 pages, 2013.

[4] V. K. Bulasara, R. Uppaluri, and M. K. Purkait, "Manufacture of nickel-ceramic composite membranes in agitated electroless plating baths," Materials and Manufacturing Processes, vol. 26, no. 6, pp. 862-867, 2011.

[5] M. P. Prabhakaran, J. R. Venugopal, T. T. Chyan et al., "Electrospun biocomposite nanofibrous scaffolds for neural tissue engineering," Tissue Engineering Part: A, vol. 14, no. 11, pp. 17871797, 2008.

[6] D. Puppi, N. Detta, A. M. Piras et al., "Development of electrospun three-arm star poly( $\varepsilon$-caprolactone) meshes for tissue engineering applications," Macromolecular Bioscience, vol. 10, no. 8, pp. 887-897, 2010.

[7] C. Mota, D. Puppi, M. Gazzarri, P. Bártolo, and F. Chiellini, "Melt electrospinning writing of three-dimensional star poly $(\epsilon-$ caprolactone) scaffolds," Polymer International, vol. 62, no. 6, pp. 893-900, 2013.

[8] N. Detta, C. Errico, D. Dinucci et al., "Novel electrospun polyurethane/gelatin composite meshes for vascular grafts," Journal of Materials Science: Materials in Medicine, vol. 21, no. 5, pp. 1761-1769, 2010.

[9] L. H. Chong, M. M. Lim, and N. Sultana, "Fabrication and evaluation of polycaprolactone/gelatin-based electrospun nanofibers with antibacterial properties," Journal of Nanomaterials, vol. 2015, Article ID 970542, 8 pages, 2015.

[10] M. Abedalwafa, F. Wang, L. Wang, and C. Li, "Biodegradable poly-epsilon-caprolactone (PCL) for tissue engineering applications: a review," Reviews on Advanced Materials Science, vol. 34, no. 2, pp. 123-140, 2013.

[11] GMIA, Gelatin Handbook, Gelatin Manufacturers Institute of America, 2012.

[12] L. Jeong and W. H. Park, "Preparation and characterization of gelatin nanofibers containing silver nanoparticles," International Journal of Molecular Sciences, vol. 15, no. 4, pp. 6857-6879, 2014.

[13] L. Van der Schueren, B. De Schoenmaker, Ö. I. Kalaoglu, and K. De Clerck, "An alternative solvent system for the steady state electrospinning of polycaprolactone," European Polymer Journal, vol. 47, no. 6, pp. 1256-1263, 2011.

[14] L. Ghasemi-Mobarakeh, M. P. Prabhakaran, M. Morshed, M.H. Nasr-Esfahani, and S. Ramakrishna, "Electrospun poly $(\varepsilon-$ caprolactone)/gelatin nanofibrous scaffolds for nerve tissue engineering," Biomaterials, vol. 29, no. 34, pp. 4532-4539, 2008.

[15] H. Shimizu, Shimizu's Textbook of Dermatology, Hokkaido University, 2007.

[16] E. J. Chong, T. T. Phan, I. J. Lim et al., "Evaluation of electrospun PCL/gelatin nanofibrous scaffold for wound healing and layered dermal reconstitution," Acta Biomaterialia, vol. 3, no. 3, pp. 321330, 2007.

[17] Y. Zhang, H. Ouyang, T. L. Chwee, S. Ramakrishna, and Z.M. Huang, "Electrospinning of gelatin fibers and gelatin/PCL composite fibrous scaffolds," Journal of Biomedical Materials Research Part B Applied Biomaterials, vol. 72, no. 1, pp. 156-165, 2005.

[18] C. Xu, F. Yang, S. Wang, and S. Ramakrishna, "In vitro study of human vascular endothelial cell function on materials with various surface roughness," Journal of Biomedical Materials Research Part A, vol. 71, no. 1, pp. 154-161, 2004.

[19] SCOGS DFs, "FDA's SCOGS database," Gelatin, Contract No. 58, 1975. 
[20] E. Schnell, K. Klinkhammer, S. Balzer et al., "Guidance of glial cell migration and axonal growth on electrospun nanofibers of poly- $\varepsilon$-caprolactone and a collagen/poly- $\varepsilon$-caprolactone blend," Biomaterials, vol. 28, no. 19, pp. 3012-3025, 2007.

[21] D. Lubasová, L. Martinová, D. Martinová, and P. Kostecká, "Cell growth on porous and non-porous polycaprolactone nanofibers," in Proceedings of the International Conference on Nano Technology (NANOCON '10), October 2010.

[22] M.-S. Khil, S. R. Bhattarai, H.-Y. Kim, S.-Z. Kim, and K.-H. Lee, "Novel fabricated matrix via electrospinning for tissue engineering," Journal of Biomedical Materials Research Part B: Applied Biomaterials, vol. 72, no. 1, pp. 117-124, 2005.

[23] Z. Ma, Z. Mao, and C. Gao, "Surface modification and property analysis of biomedical polymers used for tissue engineering," Colloids and Surfaces B: Biointerfaces, vol. 60, no. 2, pp. 137-157, 2007.

[24] R. A. Freitas, "Nanomedicine," in Basic Capabilities, vol. 1, Landes Bioscience, Georgetown, Tex, USA, 1999. 

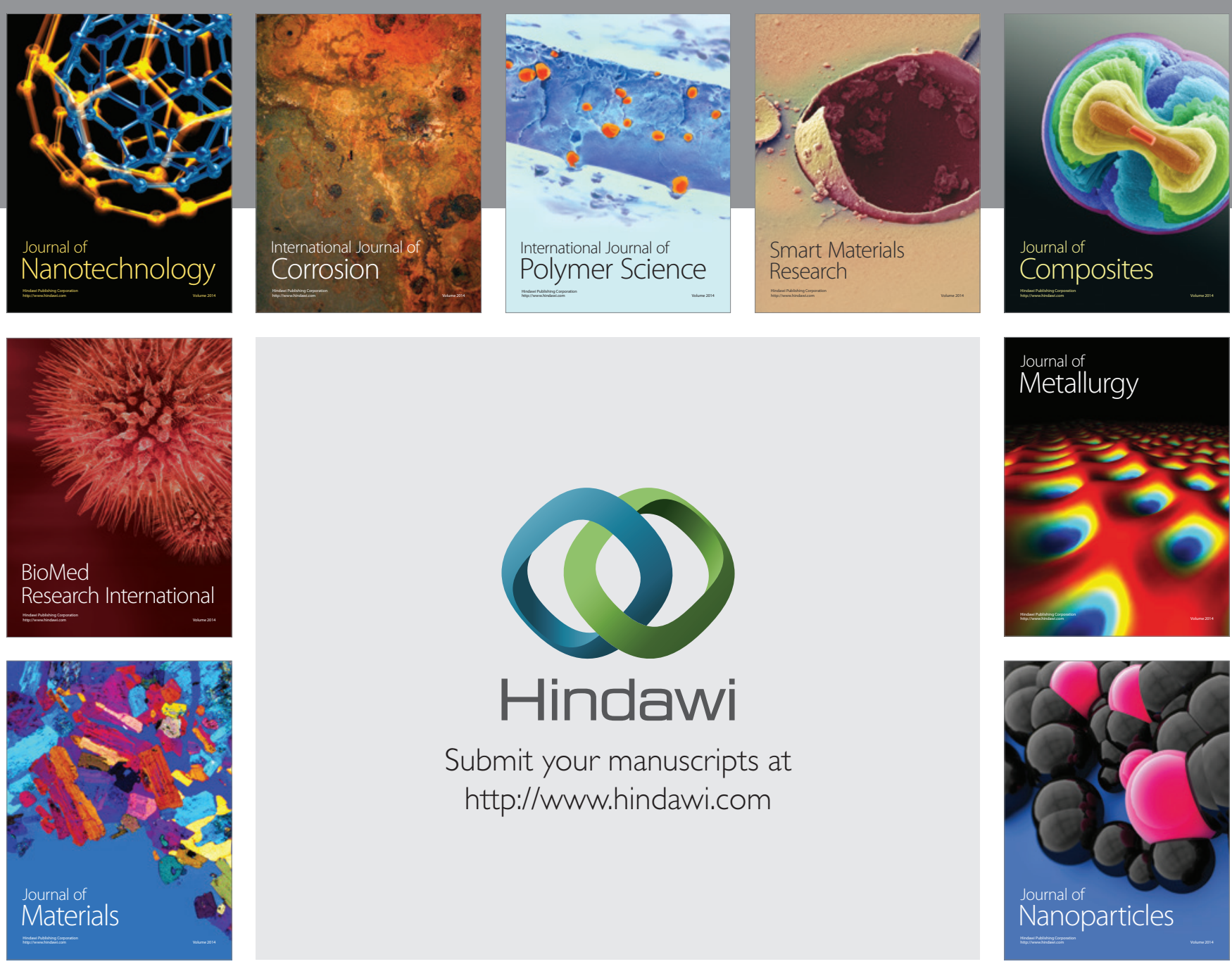

Submit your manuscripts at http://www.hindawi.com
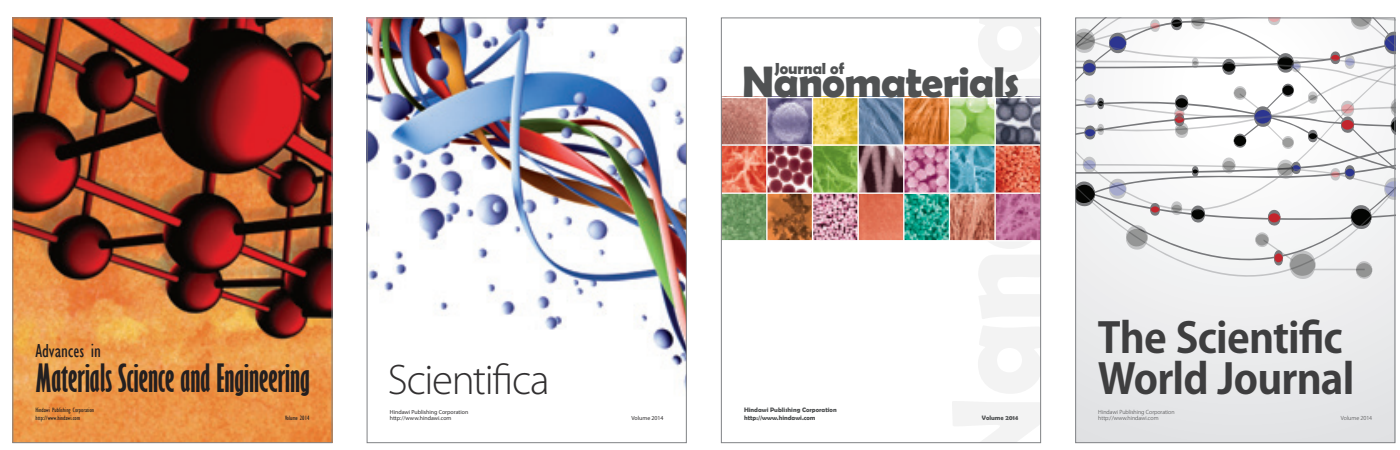

\section{The Scientific World Journal}
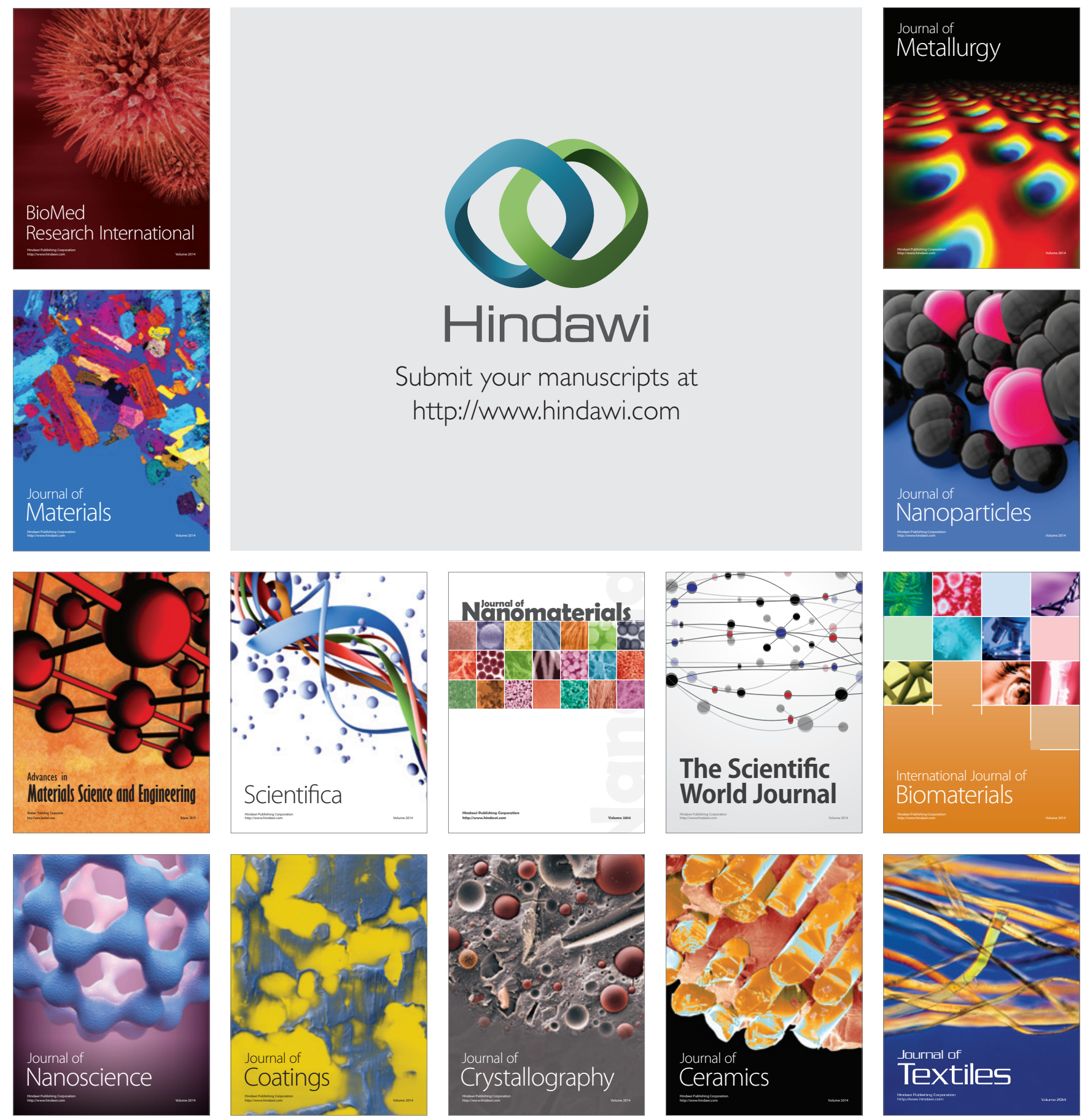